\title{
Paediatric crossword puzzle 31
}

\section{Manouri P Senanayake ${ }^{1}$, A S Athapathu ${ }^{2}$}

Sri Lanka Journal of Child Health, 2016; 45(2):143

DOI: http://dx.doi.org/10.4038/sljch.v45i2.8098

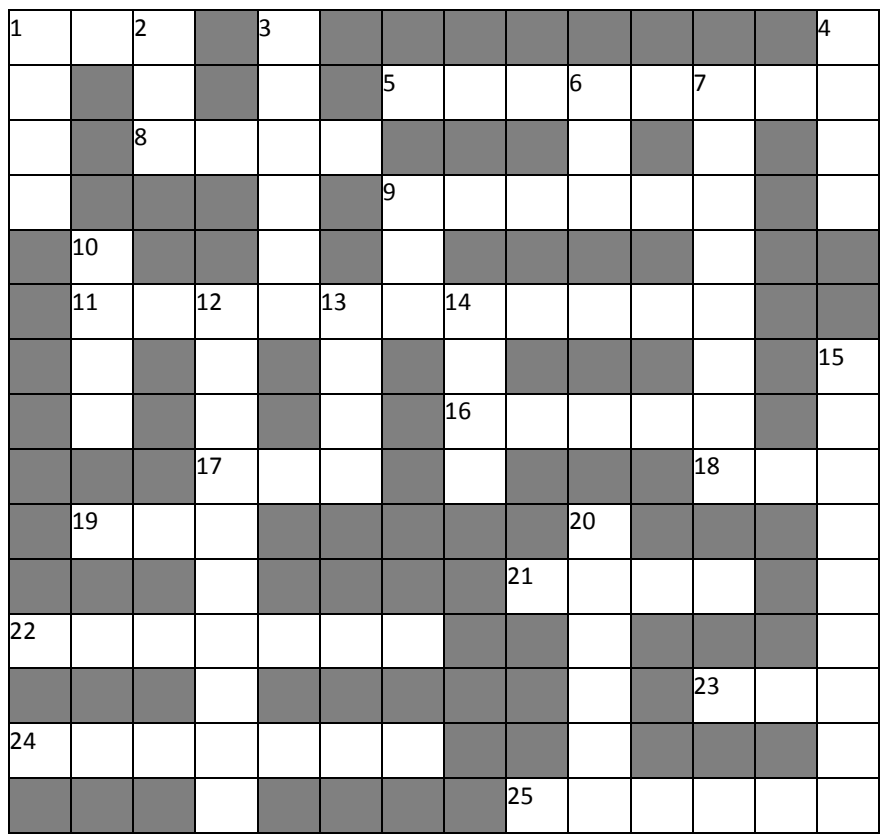

\section{ACROSS}

1. A condition named after a German Baron who was notorious for relating a

5. Disease affecting all three layers of heart

8. Synonymous with GORD

9. Sample tested for Helicobacter pylori antigen

11. Purine esterase inhibitor purified from human urine

16. Important to assess in cerebral palsy

17. A coronary artery at risk of damage in Kawasaki disease (abbrv)

18. Albinism requires ...... glasses

19. Complication of chronic constipation (abbrv)

21. Group of refugee children who are provided foster care (abbrv)

22. Repeatedly asking the same question is a feature

23. Investigation which helps diagnose epilepsy (abbrv)

24. Noticing the feelings of another

25. Prevents open neural tube defects

\section{DOWN}

1. A cytogenetic technique that detects deletions or breakages in chromosomes (abbrv)

2. Important skin sign in children in refugee camps

3. Nonsense words

4. Feature of pain threshold in autism

6. Airway, Breathing, Circulation (abbrv)

7. Vacant spells

9. Condition due to neurotoxin ingested by mother that is the commonest cause of neuro developmental delay in the West with three key diagnostic features (abbrv)

10. Mainstay of treatment in Kawasaki disease is ......... dose of ivig

12. Used in ivig resistant Kawasaki Disease

13. A co-morbidity associated with autism

14. Poor attention prevents completion of

15. Speech in autism

20. A feature considered as female genital mutilation

${ }^{1}$ Senior Professor in Paediatrics, University of Colombo, ${ }^{2}$ Intern Medical Officer, Lady Ridgeway Hospital for Children, Colombo 